\title{
The Scattering of Spin-1 Particles by Quantum Gravitational Bubbles
}

\author{
N. P. Warner \\ Department of Applied Mathematics and Theoretical Physics, University of Cambridge, Cambridge, \\ England
}

\begin{abstract}
Quantum gravitational bubbles may be used to obtain a nonperturbative approximation to the path integral of quantum gravity. There are three basic types of bubbles $C P^{2}, S^{2} \times S^{2}$, and $K 3$, and in this paper the propagation of elementary spin-1 particles in $C P^{2}$ is investigated. To date information about the propagation of particles other than scalars has been obtained by making approximations to the basic bubble types. The work presented here represents the first exact calculation. It is found that spin-1 particles scatter very strongly, particularly at low energies, which is at odds with both physical observation and the earlier work on this subject. Possible explanations for this discrepancy are offered.
\end{abstract}

\section{Introduction}

The idea of spacetime foam is that the dimensional character of the gravitational constant allows very large fluctuations of the metric and even the topology on scales less than the Planck length. Such a foamlike structure cannot be handled by ordinary perturbation theory but it might be treated by a different approximation scheme, that of gravitational bubbles $[1,2]$. In these references the authors use gravitational bubbles to calculate the behaviour of particles of spins $0, \frac{1}{2}$, and 1 in the quantum gravitational vacuum. In this paper, I shall discuss a similar calculation, the results of which are at odds with those of Hawking et al. In order to appreciate the discrepancies and possible resolutions it is necessary to discuss how one arrives at the foam model.

Consider some fields, $\phi$, propagating through a gravitational vacuum from some "in" region to some "out" region. In keeping with the usual assumptions of quantum field theory, I shall assume that there are such "in" and "out" regions which are asymptotically flat, and where the interactions and fluctuations may be turned off. The scattering amplitude for this propagation may be evaluated via the Euclidean path integral

$$
\left\langle\phi_{\text {out }} \mid \phi_{\text {in }}\right\rangle=\int \mathscr{D}[g, \phi] \exp (-I[g, \phi]),
$$


where the integral is performed over all fields $\phi$ which agree with $\phi_{\text {in }}$ and $\phi_{\text {out }}$ in the asymptotic regions, and all asymptotically Euclidean (AE) metrics [3]. The action, $I[g, \phi]$, is assumed to be Einstein, however the quantum gravitational bubble method can be used with any action, and the results will be similar to those derived here.

If we perform the integral over the fields $\phi$ for a fixed metric $g$, then we obtain the scattering amplitude for the fields propagating in that background. Therefore we may view (1.1) as being the average over all metrics of the scattering amplitudes $\left\langle\phi_{\text {out }} \mid \phi_{\text {in }}\right\rangle$ in each background, weighted by the probability that the particular background metric will appear in a vacuum fluctuation. The consequence of using a different gravitational action will be to change the weight factor given to a particular metric fluctuation, or gravitational bubble.

A useful way of handling AE metrics is by adding a point at infinity, and making a conformal transformation so as to render the metric regular everywhere. This is the analogue of the way in which Euclidean space, $R^{4}$, is compactified to $S^{4}$ in Yang-Mills theory. The reason that such compactification is helpful is that zero rest-mass particles obey equations with simple properties under conformal transformations. Even if one is dealing with massive fields, one can regard them as being effectively zero mass when compared with the Planck mass. Therefore, in practice one usually considers (1.1) to be an integral over all compact Riemannian metrics, where the fields, $\phi$, obey some suitably modified "boundary" conditions at the point of compactification.

Hawking et al. $[1,2]$ introduce what they call the finite dimensional approximation to simplify the path integral in two stages. First they assume that the fluctuations of the background involve only single Riemannian metrics, each one localized in some small region, and it is also assumed that these individual metrics are non-interacting. This is just like the dilute gas approximation of YangMills theory. The second approximation is far more drastic. The aim is to represent all Riemannian metric fluctuations by a family of simple topological units, called "bubbles," which are described by a finite number of parameters. Therefore, to compute (1.1) one obtains the scattering amplitude for each bubble, weights it with $\exp (-$ Action) and averages this over all parameter values.

Hawking et al. $[1,2]$ have observed that by taking topological sums (cutting and pasting) of combinations of $C P^{2}$, anti- $C P^{2}, K 3$, anti- $K 3$, and $S^{2} \times S^{2}$, it is possible to build a compact, simply connected 4-manifold of any Euler characteristic and Hirzebruch signature. From the generalized Poincaré conjecture it follows that any compact, simply-connected 4-manifold can be built up to homeomorphism by topological sums of these manifolds. (The four dimensional Poincaré conjecture has been proved for topological manifolds by Freedman [4].) It must be stressed that this does not mean that the metric structure can be built in this way, but simply the topology. It is suggested in [1,2] that one should use the manifolds listed above as bubbles in the finite dimensional approximation. The parameters of this family of topological units must include not only those which describe the metric on the manifold, but also the position, size and orientation of the bubble between the asymptotic "in" and "out" regions. A good physical analogy is if we have a mixture of colloids of several different oils in water. The colloids consist of droplets of oil of all different sizes. One now asks how light 
scatters off this mixture, and the ensuing calculation is formally very similar to that for these gravitational bubbles.

The similarity of this technique to the dilute gas methods of Yang-Mills theory is evident, but here the bubbles do not comprise an exhaustive list of solutions minimizing the Einstein action. The bubbles are not even gravitational instantons. Furthermore, it is very difficult to determine the correct weight factor for each bubble in the path integral. Clearly, the approximations that have been made are somewhat Draconian. However, the beauty of the gravitational bubble approach is that it is non-perturbative, and if one believes that non-trivial gravitational effects are due to topology, then one can at least get some indication of the higher order effects of the quantum gravitational vacuum by calculating how each bubble scatters elementary particles.

Hawking et al. [2] give details of their calculations of scattering massless particles by bubbles. These calculations are not very extensive because $K 3$ has an impossibly complicated family of metrics [5-7]; none of the Green's functions on $S^{2} \times S^{2}$ are known and only the scalar Green's functions on $C P^{2}$ were known until recently [8]. However, Hawking et al. made an approximation to $S^{2} \times S^{2}$ by a conformally flat manifold with conical singularities, and they used this to estimate how higher spin particles propagate in foam. Their results suggest that the scattering amplitudes are of order $\left(\frac{k_{1} \cdot k_{2}}{m_{p}^{2}}\right)^{s}$, where $s$ is the spin, $k_{1}$ and $k_{2}$ are the "in" and "out" momenta, and $m_{p}$ is the Planck mass. At first sight it may appear that such scattering would violate momentum conservation. However, what happens is that several particles must scatter in the same bubble in such a way that energy-momentum is conserved overall [2]. This amplitude shows that low energy scattering is very strong for elementary scalars, and extremely weak for higher spin elementary particles. This is not inconsistent with observation since no elementary scalars have been observed, and the higher spin effects are beyond measurement at present.

The calculation to be discussed here is for the scattering of spin-1 particles by a bubble with topology of $C P^{2}$ and a metric conformal to the standard FubiniStudy metric.

The result takes a rather different form to that in references [1,2]. Most particularly, there is very strong scattering at low energies, contrary to the usual observation that light travels in straight lines in empty space. This result does not necessarily ruin the foam model, there are some technical properties of this metric on $C P^{2}$ which may mean that it is inappropriate to use it. This will be discussed in Sect. 6 .

In Sect. 2 I shall give a cursory description of $C P^{2}$, a more complete discussion may be found in references [8] and [9]. In Sect. 3, I describe how to attach a conformal null infinity, $\mathscr{I}$, to the asymptotically Euclidean space obtained from $C P^{2}$ by sending a point to infinity, and then obtain the appropriate initial data for massless spin-1 particles. The inner products on $\mathscr{I}$ are discussed in Sect. 4 , and in particular how to use the $C P^{2}$ Green's functions to propagate from $\mathscr{I}$ to $\mathscr{I}$. The Green's function is introduced in Sect. 5, and the inner products are evaluated. Finally, the impact of my results on foam is considered in Sect. 6. 


\section{The Geometry of $C P^{2}$}

The traditional way of describing $C P^{2}$ is in the complex coordinates $\left(\zeta_{1}, \zeta_{2}\right)$, where

$$
\begin{aligned}
d s^{2} & =g_{l j} d \zeta^{i} d \bar{\zeta}^{\bar{j}} \\
& =\frac{1}{\varrho^{4}(\zeta)}\left(\varrho^{2}(\zeta) \sum_{i=1}^{2}\left|d \zeta_{i}\right|^{2}-\left|\sum_{i=1}^{2} \bar{\zeta} d \zeta^{i}\right|^{2}\right),
\end{aligned}
$$

and

$$
\varrho^{2}(\zeta)=a^{2}+\sum_{i=1}^{2}\left|\zeta_{i}\right|^{2}
$$

One requires three such coordinate patches to cover the entire manifold; the patch $\left(\zeta_{1}, \zeta_{2}\right)$ excludes a $C P^{1} \cong S^{2}$ at $\zeta_{i}=\infty$. This $C P^{1}$ is in fact a bolt [10]; that is to say the metric (2.1) has a Killing vector field which vanishes on this surface. The symmetry group of $C P^{2}$ is $\frac{\mathrm{SU}(3)}{\mathbb{Z}_{3}}$, and the stabilizer of a point is a copy of $U(2)$ [11]. Here $U(2)$ is locally isomorphic to $S U(2) \times U(1)$, and if one considers the stabilizer of the origin of the $C P^{2}, \zeta_{1}=\zeta_{2}=0$, then the factor of $\mathrm{SU}(2)$ serves to rotate the $C P^{1}$ at infinity, and the Killing vector field corresponding to the $\mathrm{U}(1)$ factor is the Killing field which vanishes on the bolt.

The metric (2.1) is Einstein-Kähler with cosmological constant $\Lambda=\frac{6}{a^{2}}$, and has Kähler form :

$$
J=i g_{i j} d \zeta^{i} \wedge d \overline{\zeta^{\bar{j}}} .
$$

For my purposes, it is more convenient to adopt real coordinates defined by

$$
\begin{aligned}
& \zeta_{1}=\left(x^{0}+i n_{j} x^{j}\right), \\
& \zeta_{2}=\left(a_{j}+i b_{j} x^{j}\right), \quad j=1,2,3,
\end{aligned}
$$

where $(a, b, n)$ is an orthonormal triad in $R^{3}$. If this triad is (left) right handed then the Weyl tensor of the $C P^{2}$ is (anti-) self-dual. The choice of $n_{j}$ and a handedness therefore determines the complex structure of the $C P^{2}$, and clearly there is an $S^{2} \times \mathbb{Z}_{2}$ worth of such structures. For the pressent I shall assume that $(a, b, n)$ is right handed; the modifications to what follows for left handed systems are trivial.

In the real coordinate system the metric is

$$
g_{\mu \nu}=\frac{a^{2}}{\varrho^{2}(x)}\left[\delta_{\mu \nu}-\frac{1}{\varrho^{2}(x)}\left(x_{\mu} x_{v}+n_{j} \eta_{\mu \sigma}^{j} \sigma^{\sigma} n_{k} \eta_{\nu \lambda}^{k} x^{\lambda}\right)\right],
$$

where $\varrho^{2}(x)=a^{2}+x^{2}$ and $\eta_{\mu \nu}^{i}$ are the self-dual 't Hooft matrices:

$$
\eta_{\mu \nu}^{1}=\left(\begin{array}{rrrr}
0 & 1 & 0 & 0 \\
-1 & 0 & 0 & 0 \\
0 & 0 & 0 & 1 \\
0 & 0 & -1 & 0
\end{array}\right), \quad \eta_{\mu \nu}^{2}=\left(\begin{array}{rrrr}
0 & 0 & 1 & 0 \\
0 & 0 & 0 & -1 \\
-1 & 0 & 0 & 0 \\
0 & 1 & 0 & 0
\end{array}\right)
$$




$$
\eta_{\mu \nu}^{3}=\left(\begin{array}{rrrr}
0 & 0 & 0 & 1 \\
0 & 0 & 1 & 0 \\
0 & -1 & 0 & 0 \\
-1 & 0 & 0 & 0
\end{array}\right)
$$

which generate right-handed rotations in $\mathrm{SO}(4)$. The Kähler form now takes the simple form:

$$
J_{\mu}{ }^{\nu}=n_{i} \eta_{\mu}^{i v}
$$

where $\eta_{\mu}^{i v}$ is numerically equal to $\eta_{\mu \nu}^{i}$. The Riemann tensor is just

$$
\begin{aligned}
R_{\alpha \beta \gamma \delta}= & \frac{\Lambda}{6}\left(g_{\alpha \gamma} g_{\beta \delta}-g_{\alpha \delta} g_{\beta \gamma}+J_{\alpha \gamma} J_{\beta \delta}\right. \\
& \left.-J_{\alpha \delta} J_{\beta \gamma}+2 J_{\alpha \beta} J_{\gamma \delta}\right) .
\end{aligned}
$$

The Hodge-DeRham Green's function satisfies

$$
\left(-\square_{x}+\Lambda\right) G_{\gamma}^{\alpha}(x, y)=\delta_{\gamma}^{\alpha} \delta(x, y)
$$

and has been obtained in $[8]$ :

$$
\begin{aligned}
G_{\alpha \mid \gamma}(x, y) & =\frac{1}{2}\left(\delta_{\gamma}^{\beta} \delta_{\gamma}^{\delta}+\eta^{\beta}{ }_{\alpha} \eta_{\gamma}^{\delta}\right)\left[f_{1}(L) \frac{\partial^{2} L}{\partial x^{\beta} \partial y^{\delta}}+f_{2}(L) \frac{\partial L}{\partial x^{\beta}} \frac{\partial L}{\partial y^{\delta}}\right] \\
f_{1}(L) & =\frac{1}{4 \pi^{2}}\left[\frac{1}{(1-L)}+\frac{1}{2 L} \log (1-L)\right] \\
f_{2}(L) & =-\frac{1}{8 \pi^{2}}\left[\frac{1}{L(1-L)}+\frac{1}{L^{2}} \log (1-L)\right] \\
L(x, y) & =\frac{\left|a^{2}+x_{\mu} y^{\mu}+i n_{j} \eta_{\mu \nu}^{j} x^{\mu} y^{\mu}\right|^{2}}{\varrho^{2}(x) \varrho^{2}(y)}
\end{aligned}
$$

where $\alpha$ and $\gamma$ are indices at $x$ and $y$ respectively and the "l" between $\alpha$ and $\gamma$ is a notation adopted to separate indices belonging to different endpoints.

In order to set up the initial data, I will need the parallel propagator [12] for geodesics on $C P^{2}$. That is, the two point vector $\tau_{\gamma}^{\alpha}(x, y)$ which satisfies the equation of parallel transport along the geodesic from $y$ to $x$, and also has $\tau^{\alpha}{ }_{\gamma}\left(x, x=\delta_{\gamma}^{\alpha}\right.$. The geodesic structure is nearly trivial, and it is no difficult task to show that

$$
\tau_{\gamma}^{\alpha}(x, 0)=\frac{\varrho(x)}{a}\left[\delta_{\gamma}^{\alpha}+\frac{1}{a(a+\varrho(x))}\left(x^{\alpha} x_{\gamma}+n_{j} \eta_{\sigma}^{j \alpha} x^{\sigma} n_{k} \eta_{\varrho \gamma}^{k} x^{\varrho}\right)\right] .
$$

Finally, for convenience I shall adopt the notation, $\eta_{\mu \nu}=n_{k} \eta_{\mu \nu}^{k}$, and

$$
x_{v}^{\prime}=\eta_{\mu v} x^{\mu} \quad \text { and } \quad y_{v}^{\prime}=\eta_{v \mu} y^{\mu}
$$

(Note the change of indices.) 


\section{Null Infinity and the Data}

In order to consider the scattering of particles by a $C P^{2}$ bubble one must first go to an asymptotically Euclidean space, $M$, obtained from $C P^{2}$ by sending the origin to infinity and conformally transforming, the metric to

$$
g_{\mu \nu}^{\prime}=\Omega^{2} g_{\mu \nu} .
$$

A neighbourhood of the origin of $C P^{2}$ is mapped to the asymptotic region of $M$, and one may identify the sphere at infinity of $M$ with the unit sphere in the tangent bundle at the origin of $C P^{2}$. Thus the asymptotic region of $M$ may be complexified and a Lorentzian section can be defined in the tangent plane at infinity. This Lorentzian section clearly cannot be extended into the manifold since the Weyl tensor is self dual. However, asymptotically Lorentzian sections are sufficient since the physically measurable quantities, the data, are defined there only. We may therefore attach an $\mathscr{I}$ to $M$, and this may be conformally mapped back onto $C P^{2}$.

Obviously there are many possible choices of conformal factor in the foregoing construction. Here $\Omega$ is fixed by requiring that $R\left(g_{\mu \nu}^{\prime}\right)=0$ and that

$$
R_{\mu v} l^{\mu} l^{v}=0 \text { on } \mathscr{I}
$$

where $l^{\mu}$ is the null vector tangent to $\mathscr{I}$. Then one obtains

$$
\Omega=b^{2}\left(\frac{1}{a^{2}}+\frac{1}{x^{2}}\right)
$$

where $b$ is a constant scale factor.

The gravitational action of $g_{\mu \nu}^{\prime}$ comes purely from the boundary term, and is given by

$$
I_{0}=\frac{\pi}{4} b^{4} / a^{2}
$$

The metric $g_{\mu \nu}^{\prime}$ defined by (3.1) and (3.3) is asymptotically Euclidean as advertised. The Weyl tensor has the correct asymptotic behaviour as one approaches the sphere at infinity. However, on the Lorentzian section of the asymptotic region, the space-time is not flat at $\mathscr{I}$; specifically, the Weyl tensor does not vanish.

In order to study the structure of $\mathscr{I}$ it is simpler to work in $C P^{2}$. Under the conformal map (3.3), $\mathscr{I}$ is mapped to a complex cone through the origin of $C P^{2}$, described by

$$
x^{\mu}=l^{\mu} v^{\prime}
$$

where $l^{\mu}$ is some null vector at $x=0$. Equation (3.5) describes a geodesic, and $v^{\prime}$ is an affine parameter. Let $l^{\mu}, p^{\mu}, f^{\mu}$, and $\tilde{f}^{\mu}$ be a null tetrad at 0 , such that $f_{\mu} \tilde{f}^{\mu}$ $=-l^{\mu} p_{\mu}=1$. Define a null tetrad $l^{\mu}, n^{\mu}, m^{\mu}, \tilde{m}^{\mu}$ on $\mathscr{I}$ by parallel transport along the geodesic described by (3.5). It should be pointed out that the tilda over $\tilde{f}_{\mu}$ and $\tilde{m}_{\mu}$ does not indicate that these vectors are the complex conjugates of $f_{\mu}$ and $m_{\mu}$. The tilda is intended to denote that these four quantities are the analytic continuations of vectors which are complex conjugates in Lorentz space. 
From (2.14) and (2.15) it follows that

$$
\begin{aligned}
m^{\mu} & =f^{\mu}+\frac{1}{2 a^{2}}\left(x^{\prime} \cdot f\right) x^{\prime \mu}, \\
\tilde{m}^{\mu} & =\tilde{f}^{\mu}+\frac{1}{2 a^{2}}\left(x^{\prime} \cdot \tilde{f}\right) x^{\prime \mu}=\tilde{f}^{\mu}, \\
n^{\mu} & =p^{\mu}+\frac{1}{2 a^{2}}\left[(x \cdot p) x^{\mu}+\left(x^{\prime} \cdot p\right) x^{\prime \mu}\right] \\
& =p^{\mu}+\frac{1}{2 a^{2}}\left[v^{\prime} x^{\mu}+\left(x^{\prime} \cdot f\right) x^{\prime \mu}\right],
\end{aligned}
$$

where the dot product denotes the inner product with respect to the flat, diagonal Euclidean or Lorentzian metric. Self duality of $\eta_{\mu \nu}$ implies that $\left(x^{\prime} \cdot \tilde{f}\right)=0$ and $\left(x^{\prime} \cdot p\right)$ $=\left(x^{\prime} \cdot f\right)$. Therefore $\tilde{m}^{\mu}$ propagates trivially, and $m^{\mu}$ twists ; this is a manifestation of the fact that the anti-self-dual part of the Weyl tensor vanishes.

One can define local coordinates on $\mathscr{I}$ by normalizing $l^{\mu}$ to have a component of $\frac{1}{2}$ in some timelike direction, and setting up polar coordinates $(\theta, \phi)$ to describe the direction of $l^{\mu}$. In this system I shall take

$$
\begin{aligned}
& f^{v} \frac{\partial}{\partial x^{v}}=\frac{\sqrt{2}}{v^{\prime}}\left(\frac{\partial}{\partial \theta}+\frac{i}{\sin \theta} \frac{\partial}{\partial \phi}\right), \\
& \tilde{f}^{v} \frac{\partial}{\partial x^{v}}=\frac{\sqrt{2}}{v^{\prime}}\left(\frac{\partial}{\partial \theta}-\frac{i}{\sin \theta} \frac{\partial}{\partial \phi}\right) .
\end{aligned}
$$

Then the relevant Newman-Penrose coefficients for $\mathscr{I}$ are:

$$
\begin{array}{cc}
\varrho=l_{\mu ; v} m^{\mu} \tilde{m}^{v}=\frac{1}{v^{\prime}} ; & \tilde{\varrho}=l_{\mu ; v} \tilde{m}^{\mu} m^{v}=\frac{1}{v^{\prime}}, \\
\sigma=l_{\mu ; v} m^{\mu} m^{v}=-\frac{1}{a^{2} v^{\prime}}\left(x^{\prime} \cdot f\right)^{2} ; & \tilde{\sigma}=l_{\mu ; v} \tilde{m}^{\mu} \tilde{m}^{v}=0, \\
\alpha+\tilde{\beta}=l_{\mu ; v} n^{\mu} \tilde{m}^{v}=0 ; & \alpha-\tilde{\beta}=-m_{\mu ; v} \tilde{m}^{\mu} \tilde{m}^{v}=\frac{1}{v^{\prime}} \sqrt{2} \cot \theta, \\
\tilde{\alpha}+\beta=l_{\mu ; v} n^{\mu} m^{\mu}=-\frac{3}{2 a^{2}}\left(x^{\prime} \cdot f\right)^{2}, & \\
\tilde{\alpha}-\beta & =m_{\alpha ; \beta} \tilde{m}^{\alpha} m^{\beta}=\frac{\sqrt{2} \cot \theta}{v^{\prime}}\left[1-\frac{1}{2 a^{2}}\left(x^{\prime} \cdot f\right)^{2}\right]+\frac{3}{2 a^{2}}\left(x^{\prime} \cdot f\right)\left(\eta_{\mu \nu} \tilde{v}^{\mu} f^{v}\right) .
\end{array}
$$

Observe that $\mathscr{I}$ has right handed shear $\sigma$, but that the left handed shear, $\tilde{\sigma}$, vanishes. Also note that as $v^{\prime} \rightarrow \infty, \sigma=-\frac{v^{\prime}}{a^{2}}\left(\eta_{\mu \nu} \mu^{\mu} f^{\mu}\right) \rightarrow \infty$, and hence $\mathscr{I}$ has singular shear as one approaches the complexified neighbourhood of the bolt. If 
one approaches the bolt even slightly away from a null direction, the factors of $\frac{1}{a^{2}+x^{2}}$ serve to prevent divergences in the shear and Weyl tensors. On null directions these factors become $\frac{1}{a^{2}}$. Hence the null cone through the origin is peculiar in that it blows up at the bolt and yet the interior and exterior of the cone are well behaved as $x \rightarrow \infty$. This is probably a major factor contributing to the discrepancy between my scattering calculations and expectations.

The null hypersurface initial value problem is treated very thoroughly in [13]. On a null surface the field equations do not yield constraints on the data, but determine the normal derivatives of the data out of the surface from the data on the surface. For a Maxwell field one needs to specify only $F_{\mu \nu} l^{\mu} m^{\nu}$ and $F_{\mu \nu} l^{\mu} \tilde{m}^{\nu}$. Hawking et al. [2] have shown that if one conformally maps a flat $\mathscr{I}$ to the origin in a similar manner to the foregoing analysis, then the data for plane waves of momentum $k^{\mu}$ in the asymptotic region becomes:

$$
F_{\mu \nu} l^{\mu} m^{\nu}=0 ; \quad F_{\mu \nu} l^{\mu} \tilde{m}^{v}=N \frac{e^{i \omega b^{2} / v^{\prime}}}{v^{\prime 3}} \frac{\delta\left(\theta-\theta_{k}\right) \delta\left(\phi-\phi_{k}\right)}{\sin \theta}
$$

for self dual data, and

$$
F_{\mu \nu} l^{\mu} \tilde{m}^{\nu}=0 ; \quad F_{\mu \nu} l^{\mu} \tilde{m}^{\nu}=N \frac{e^{i \omega b^{2} / v^{\prime}}}{v^{\prime 3}} \frac{\delta\left(\theta-\theta_{k}\right) \delta\left(\phi-\phi_{k}\right)}{\sin \theta}
$$

for an anti-self-dual field. Here $N$ is a normalization constant, $\theta_{k}$ and $\phi_{k}$ are the angular directions of the plane waves on $\mathscr{I}$, and $\omega=-k^{\mu} n_{\mu}$. It is elementary to show from the field equations that the data must also satisfy

$$
F_{\mu \nu} l^{\mu} n^{\nu}= \pm F_{\mu \nu} m^{\mu} \tilde{m}^{\nu}
$$

with + or - for self-dual or anti-self-dual data respectively.

This may be generalized to the case of $C P^{2}$, where $\mathscr{I}$ is not flat, by simply dictating that in (3.9) and (3.10) $l^{\mu}, m^{\mu}, \tilde{m}^{\mu}$, and $n^{\mu}$ must be a parallel transported tetrad. Naturally (3.11) remains true.

To construct a gauge potential for this data on $C P^{2}$ I shall take the light cone gauge

$$
A_{\mu} l^{\mu}=0
$$

if and only if

$$
A_{\mu}=h_{1} \tilde{m}_{\mu}+h_{2} m_{\mu}-h_{3} l_{\mu}
$$

Then

$$
\begin{aligned}
& F_{\mu \nu} l^{\mu} m^{v}=-\frac{1}{v^{\prime}} \frac{\partial}{\partial v^{\prime}}\left(v^{\prime} h_{1}\right)+\frac{1}{a^{2} v^{\prime}}\left(x^{\prime} \cdot f\right)^{2} h_{2}, \\
& F_{\mu \nu} l^{\mu} \tilde{m}^{v}=-\frac{1}{v^{\prime}} \frac{\partial}{\partial v^{\prime}}\left(v^{\prime} h_{2}\right),
\end{aligned}
$$

where I have used (3.8). 
For anti-self-dual data we may take

$$
\begin{aligned}
& h_{1}=-\frac{i N}{\omega b^{2}} \frac{e^{i \omega b^{2} / v^{\prime}}}{v^{\prime}} \frac{\delta\left(\theta-\theta_{k}\right) \delta\left(\phi-\phi_{k}\right)}{\sin \theta}, \\
& h_{2}=0
\end{aligned}
$$

and for self-dual data

$$
\begin{gathered}
h_{2}=\frac{-i N}{\omega b^{2}} \frac{e^{i \omega b^{2} / v^{\prime}}}{v^{\prime}} \frac{\delta\left(\theta-\theta_{k}\right) \delta\left(\phi-\phi_{k}\right)}{\sin \theta}, \\
\frac{\partial}{\partial v^{\prime}}\left(v^{\prime} h_{1}\right)=\frac{1}{a^{2}}\left(x^{\prime} \cdot f\right)^{2} h_{2} .
\end{gathered}
$$

The choice of $h_{3}$ is undetermined at present, this represents the remaining gauge freedom. $A_{\mu} l^{\mu}$ corresponds to the fixed gauge freedom of the background, and $h_{3}=A_{\mu} n^{\mu}$ contains the information about gauge propagating from $\mathscr{I}$ to $\mathscr{I}$. From (3.11) it can be shown that

$$
\nabla_{\mu} A^{\mu}= \begin{cases}-2\left[\frac{\partial h_{3}}{\partial v^{\prime}}-A_{\mu ; v} \tilde{m}^{\mu} m^{v}\right] & (\text { self-dual) } \\ -2\left[\frac{\partial h_{3}}{\partial v^{\prime}}-A_{\mu ; v} m^{\mu} \tilde{m}^{v}\right] & \text { (anti-self-dual) }\end{cases}
$$

Hence I may choose $h_{3}$ by the further gauge condition $\nabla_{\mu} A^{\mu}=0$. The initial data is summarized in Table 1.

Table 1. Initial Data for Maxwell Fields on the Null Cone through the Origin of $\mathrm{CP}^{2}$

Gauge Conditions:

$$
\begin{aligned}
A_{\mu} l^{\mu} & =0, \nabla_{\mu} A^{\mu}=0 \\
A_{\mu} & =h_{1} \tilde{m}_{\mu}+h_{2} m_{\mu}-h_{3} l_{\mu}
\end{aligned}
$$

Anti-Self-Dual-Data

$$
\begin{aligned}
F_{\mu \nu} l^{\mu} m^{v} & =N \frac{e^{i \omega b^{2} / v^{\prime}}}{v^{\prime 3}} \frac{\delta\left(\theta-\theta_{k}\right) \delta\left(\phi-\phi_{k}\right)}{\sin \theta} \\
F_{\mu \nu} l^{\mu} \tilde{m}^{v} & =0, \quad F_{\mu v} l^{\mu} n^{v}=F_{\mu v} \tilde{m}^{\mu} m^{v} \\
h_{1} & =\frac{-i N}{\omega b^{2}} \frac{e^{i \omega b^{2} / v^{\prime}}}{v^{\prime}} \frac{\delta\left(\theta-\theta_{k}\right) \delta\left(\phi-\phi_{k}\right)}{\sin \theta} \\
h_{2} & =0 \\
\frac{1}{v^{\prime}} \frac{\partial}{\partial v^{\prime}}\left(v^{\prime} h_{3}\right) & =\left(h_{1} \tilde{m}_{\mu}\right)_{; v} m^{\mu} \tilde{m}^{v}
\end{aligned}
$$


Self-Dual-Data

$$
\begin{aligned}
F_{\mu \nu} l^{\mu} \tilde{m}^{v} & =N \frac{e^{i \omega b^{2} / v^{\prime}}}{v^{\prime}} \frac{\delta\left(\theta-\theta_{k}\right) \delta\left(\phi-\phi_{k}\right)}{\sin \theta} \\
F_{\mu \nu} l^{\mu} m^{\nu} & =0, \quad F_{\mu \nu} l^{\mu} n^{v}=-F_{\mu \nu} \tilde{m}^{\mu} m^{\nu} \\
\frac{\partial}{\partial v^{\prime}}\left(v^{\prime} h_{1}\right) & =\frac{1}{a^{2}}\left(x^{\prime} \cdot f\right)^{2} h_{2} \\
h_{2} & =-\frac{i N}{\omega b^{2}} \frac{e^{i \omega b^{2} / v^{\prime}}}{v^{\prime}} \frac{\delta\left(\theta-\theta_{k}\right) \delta\left(\phi-\phi_{k}\right)}{\sin \theta} \\
\frac{1}{v^{\prime}} \frac{\partial}{\partial v^{\prime}}\left(v^{\prime} h_{3}\right) & =\left(h_{2} m_{\mu}\right)_{;} \tilde{m}^{\mu} m^{v} .
\end{aligned}
$$

\section{The Inner Product}

In order to calculate the scattering amplitude one must set up a quantized Maxwell theory in the neighbourhood of $\mathscr{I}$. The Cauchy data obtained in the previous section provide a set of mode functions with which to define particle states. The inner product between mode functions on a hypersurface, $\Sigma$, is

$$
I_{\Sigma}\left(A_{1}, A_{2}\right)=i \int_{\Sigma} A_{1}^{\mu} F_{2 \mu \nu}-A_{2}^{\mu} F_{1 \mu \nu} d \Sigma^{\nu}
$$

where $A_{i}$ is a gauge potential for $F_{i}$. Each term of (4.1) is separately gauge invariant, as can be verified by integration by parts within the hypersurface. The inner product can be used to obtain inner products involving the Maxwell field operators. Annihilation and creation operators are then defined on $\mathscr{I}$, and the Cauchy data appropriate to a one particle state may be obtained. Normalization is fixed by the commutation relations. The details may be found in [2]; here I shall merely state that the Cauchy data is to be taken as in the last section, with normalization constant $N=2 b^{2} \pi^{-1 / 2}$.

Consider a gauge potential satisfying Maxwell's equations in a region $V$ with boundary $\Sigma$. Then

$$
-\square A_{\mu}+R_{\mu}{ }^{v} A_{v}+\nabla_{\mu}\left(\nabla^{v} A_{v}\right)=0
$$

Define a field

$$
B^{\alpha}(x)=\int_{V} A^{\mu}(y) \mathscr{L}_{y} G^{\alpha}{ }_{\mu}(x, y)-G^{\alpha}{ }_{\mu}(x, y) \mathscr{L}_{y} A^{\mu}(y) d V(y),
$$

where $\mathscr{L}_{y}$ is the Hodge-DeRham operator at $y$ :

$$
\mathscr{L}_{y} A_{\mu}=-\square A_{\mu}+R_{\mu}{ }^{\nu} A_{\nu},
$$

and $G^{\alpha}{ }_{\mu}(x, y)$ is the Hodge-DeRham propagator satisfying (2.9). Using these facts in (4.3)

$$
\begin{aligned}
B^{\alpha}(x)= & A^{\alpha}(x)-\int_{V}\left(\nabla_{y}^{\mu} G_{\mu}^{\alpha}(x, y)\left(\nabla_{v} A^{v}\right) d V(y)\right. \\
& +\int_{\Sigma} G_{\mu}^{\alpha}(x, y)\left(\nabla_{v} A^{v}\right) d \Sigma^{\mu}(y) .
\end{aligned}
$$


However using Green's theorem in (4.3)

$$
B^{\alpha}(x)=-\int_{\Sigma} A^{\mu}(y) \overleftrightarrow{\nabla}_{\nu} G_{\mu}^{\alpha}(x, y) d \Sigma^{\nu}(y) .
$$

Hence

$$
\begin{aligned}
A^{\alpha}(x)= & -\int_{\Sigma} A^{\mu}(y) \overleftrightarrow{\nabla}_{\nu} G_{\mu}^{\alpha}(x, y)+G^{\alpha}{ }_{\nu}(x, y)\left(\nabla_{\mu} A^{\mu}\right) d \Sigma^{\nu}(y) \\
& +\int_{V}\left(\nabla^{\mu} G_{\mu}^{\alpha}(x, y)\left(\nabla_{v} A^{\nu}\right) d V(y) .\right.
\end{aligned}
$$

It is elementary to show that

$$
\nabla_{y}^{\mu} G_{\mu}^{\alpha}(x, y)=-\nabla_{x}^{\alpha} G(x, y),
$$

where

$$
-\square_{x} G(x, y)=\delta(x, y)-c .
$$

The constant $c$ is subtracted because it is a zero mode of $-\square$. Consequently the last term of (4.4) is pure gauge. Anti-symmetry may be restored to (4.4) by adding another pure gauge term. Therefore

$$
\begin{aligned}
A^{\alpha}(x)= & -\int_{\Sigma} A^{\mu}(y) \overleftrightarrow{\nabla}_{\nu} G_{\mu}^{\alpha}(x, y)+G_{\nu}^{\alpha}(x, y)\left(\nabla_{\mu} A^{\mu}\right) \\
& -\left(\nabla_{y}^{\mu} G_{\mu}^{\alpha}(x, y)\right) A_{\nu}(y) d \Sigma^{\nu}(y) \\
& + \text { pure gauge terms } .
\end{aligned}
$$

It should be noted that in order to derive this I assumed that the hypersurface $\Sigma$ bounded the volume $V$; in my case $\Sigma$ is made up of two null hypersurfaces. However it may be shown that the Cauchy data is sufficient if I take one such surface. From now on I shall assume that $\Sigma$ is a null hypersurface.

Equation (4.5) may be integrated by parts within the hypersurface to yield

$$
\begin{aligned}
& A^{\alpha}=-2 \int_{\Sigma} A^{\mu}(y) G_{[\mu ; v]}^{\alpha}(x, y)-G^{\alpha \mid \mu}(x, y) A_{[\mu ; v]} d \Sigma^{\nu}(y) \\
& \quad+\text { gauge terms }
\end{aligned}
$$

and therefore the inner product (4.1) may be used with the Hodge-DeRham Green's function to propagate the Maxwell potential up to gauge transformations. The $S$-matrix element for a spin-1 field $A_{1}$ on $\Sigma_{1}$ propagating to a spin-1 field $A_{2}$ on $\Sigma_{2}$ may now be written up to a phase factor as:

$$
S=I_{\Sigma_{2}}\left(A_{2}, I_{\Sigma_{1}}\left(G, A_{1}\right)\right),
$$

where the inner product $I_{\Sigma}$ in (4.7) is defined by (4.1).

There is a further simplification that may be made before explicitly calculating the integral. Consider the expression

$$
J=2 \int_{\Sigma} A^{\alpha} B_{[\alpha, \beta]}-A_{[\alpha, \beta]} B^{\alpha} d \Sigma^{\beta},
$$

where $\Sigma=\mathscr{I}, A^{\alpha}$ is the Cauchy data in Table 1 and $B^{\alpha}$ is an arbitrary vector field. With the coordinates chosen in Sect. 3 , the volume element is

$$
d \Sigma^{\beta}=\frac{1}{4} v^{\prime 2} l^{\beta} d v^{\prime} \sin \theta d \theta d \phi,
$$


where $l^{\beta} \frac{\partial}{\partial x^{\beta}}=\frac{\partial}{\partial v^{\prime}}$ is a null generator on $\mathscr{I}$. Suppose, for the sake of simplicity, that $A^{\alpha}(x)$ represents an anti-self-dual Maxwell field, then

$$
\begin{aligned}
& 2 \int_{\Sigma} A_{[\alpha, \beta]} B^{\alpha} d \Sigma^{\beta} \\
& \quad=\int_{\Sigma} 2 A_{[\alpha ; \beta]}\left[-l^{\beta} n^{\alpha}\left(l_{\gamma} B^{\gamma}\right)+m^{\alpha} l^{\beta}\left(\tilde{m}_{\gamma} B^{\gamma}\right)\right] \frac{v^{\prime 2}}{4} d v^{\prime} \sin \theta d \theta d \phi .
\end{aligned}
$$

However

$$
2 A_{[\alpha ; \beta]} n^{\alpha} l^{\beta}=2 A_{[\alpha ; \beta]} \tilde{m}^{\alpha} m^{\beta}=\left(h_{1} \tilde{m}_{\alpha}\right)_{; \beta} \tilde{m}^{\beta} m^{\alpha}
$$

and

$$
\left.2 A_{[\alpha ; \beta]}\right]^{\alpha} m^{\beta}=-\left(\frac{\partial}{\partial v^{\prime}}+\frac{1}{v^{\prime}}\right) h_{1} .
$$

Substituting this into (4.9) and integrating both terms by parts, using (3.7) and (3.8), one obtains

$$
\int_{\Sigma} h_{1}\left[-\left(\frac{\partial}{\partial v^{\prime}}+\frac{1}{v^{\prime}}\right)\left(B_{\alpha} \tilde{m}^{\alpha}\right)+\tilde{m}^{\nu} \frac{\partial}{\partial x^{\nu}}\left(l^{\nu} B_{\gamma}\right)\right] \frac{v^{\prime 2}}{4} d v^{\prime} \sin \theta d \theta d \phi .
$$

Finally using $\varrho=\frac{1}{v^{\prime}}$ and that $\Gamma_{\alpha \beta}^{\sigma} l^{\alpha} \tilde{m}^{\beta}=0$ for all connection coefficients, the immediately preceding expression reduces to

$$
\begin{gathered}
\int_{\Sigma} h_{1}\left[B_{\alpha ; \beta} l^{\alpha} \tilde{m}^{\beta}-B_{\beta ; \alpha} l^{\alpha} \tilde{m}^{\beta}\right] \frac{v^{\prime 2}}{4} d v^{\prime} \sin \theta d \theta d \phi \\
=-2 \int_{\Sigma} h_{1} B_{[\alpha, \beta]} \tilde{m}^{\alpha} d \Sigma^{\beta} .
\end{gathered}
$$

Hence

$$
J=4 \int_{\Sigma} h_{1} B_{[\alpha, \beta]} \tilde{m}^{\alpha} d \Sigma^{\beta} .
$$

The self-dual case is more complicated since $\mathscr{I}$ is not flat in this sector, but the result is just the same

$$
J=4 \int_{\Sigma} h_{2} B_{[\alpha, \beta]} m^{\alpha} d \Sigma^{\beta}
$$

These expressions are very satisfactory because the integrands manifestly extract the self-dual or anti-self-dual parts of $B_{\alpha}$, and contain only the primary data, $h_{1}$ and $h_{2}$ in each case respectively.

The scattering amplitude may now be written

$$
S=16 \int_{\Sigma_{1}} \int_{\Sigma_{2}} G_{[\alpha, \beta] \mid[\gamma, \delta]} q_{1}^{\alpha} q_{2}^{\gamma} j_{1}(x) j_{2}(y) d \Sigma^{\beta}(x) d \Sigma^{\delta}(y),
$$

where $j_{1}(x) q_{1}^{\alpha}$ and $j_{2}(x) q_{2}^{\alpha}$ are either $h_{1}(x) \tilde{m}^{\alpha}$ or $h_{2}(x) m^{\alpha}$ depending upon the nature of the initial and final data. 


\section{The Scattering Amplitudes}

The Maxwell equations decouple into the self-dual and anti-self-dual parts

$$
F_{; \nu}^{\mu \nu}=0 \Leftrightarrow \nabla_{A^{\prime}}{ }^{A} \phi_{A B}=0 \text { and } \nabla_{A}^{A^{\prime}} \phi_{A^{\prime} B^{\prime}}=0 .
$$

Consequently (anti-) self-dual data can only propagate to (anti-) self-dual data. Hence the spinor form of $G_{[\alpha, \beta] \mid[\gamma, \delta]}$ should have one pair of self-dual indices and one pair of anti-self-dual indices. This is true in $C P^{2}$, save for one small detail. The Kähler form (2.3) yields a self-dual Maxwell field, $F=2 J$, such that $F^{\mu v}{ }_{; v}=0$; furthermore, this Maxwell field is a non-trivial co-cycle, that is, it has no globally defined gauge potential. The presence of this topologically non-trivial zero mode gives rise to complications in the form of $G_{[\alpha, \beta] \mid[\gamma, \delta]}$, even though it cannot contribute to $G_{\alpha \mid \gamma}$. Indeed, it may be shown directly from (2.10) that

$$
J^{\alpha \beta}(x) J^{\gamma \delta}(y) G_{[\alpha, \beta] \mid[\gamma, \delta]}(x, y)=-\frac{4}{\pi^{2}}, \quad(x \neq y) .
$$

To discover the origin of this parity violation, consider the expression for $G_{\alpha \mid \gamma}(x, y)$ in terms of eigenmodes:

$$
G_{\alpha \mid \gamma}(x, y)=\sum_{k} \frac{\psi_{\alpha}^{k}(x) \psi_{\gamma}^{k}(y)}{\lambda_{k}},
$$

where $\psi_{\alpha}^{k}$ are the eigenvectors of the Hodge DeRham operator, and $\lambda_{k}$ are the eigenvalues. These eigenvectors may be obtained in terms of scalar eigenmodes and gauge covariant constant spinors $[8,14]$. From this one can show that for $x \neq y$ the only parity violating term contained in $G_{[\alpha, \beta][\gamma, \delta]}$ is

$$
-\frac{1}{4 \pi^{2}} J_{\alpha \beta}(x) J_{\gamma \delta}(y) .
$$

However, in addition, there are some terms involving the $\delta$-function, $\delta(x, y)$. All these terms are also parity violating. The $\delta$-function contributions are precisely what are needed in the $S$-matrix integral in order to cancel the term (5.2).

Observe that

$$
\begin{aligned}
\int \psi_{[\alpha, \beta]} J^{\alpha \beta} d^{4} y & =\int\left(\psi_{\alpha} J^{\alpha \beta}\right)_{; \beta} d^{4} y \\
& =0 .
\end{aligned}
$$

Hence

$$
\int G_{[\alpha, \beta][\gamma, \delta]}(x, y) J^{\gamma \delta} d^{4} y=0,
$$

which establishes that these $\delta$-functions serve to do the necessary cancellations in the case of sources in Euclidean space. The case of propagation from $\mathscr{I}$ to $\mathscr{I}$ is more complicated since one is now integrating a $\delta$-function over two threedimensional surfaces. However there will be a non-zero contribution from the points where the initial and final hypersurfaces intersect, and this must cancel with the contribution arising from (5.2). The simplest way to proceed, therefore, is to compute $G_{[\alpha, \beta][\gamma, \delta]}$ and neglect all parity violating terms of this nature. 
One can simplify the calculations by removing some gauge terms from $G_{\alpha \mid \gamma}$. Define

$$
\begin{aligned}
E_{\alpha \mid \gamma} & =f_{1}(L) \frac{\partial^{2} L}{\partial x^{\alpha} \partial y^{\gamma}}+f_{2}(L) \frac{\partial L}{\partial x^{\alpha}} \frac{\partial L}{\partial y^{\gamma}}, \\
F_{\alpha \mid \gamma} & =\frac{1}{4 \pi^{2}} \frac{1}{(1-L)} \frac{\partial^{2} L}{\partial x^{\alpha} \partial y^{\gamma}}, \\
H_{\alpha \mid \gamma} & =E_{\alpha \mid \gamma}-G_{\alpha \mid \gamma} .
\end{aligned}
$$

Observe that

$$
\left(L \frac{\partial^{2} L}{\partial x^{\sigma} \partial y^{\varrho}}-\frac{\partial L}{\partial x^{\sigma}} \frac{\partial L}{\partial y^{\varrho}}\right)\left(\delta_{\alpha}^{\sigma} \delta_{\gamma}^{\varrho}-\eta_{\alpha}^{\sigma} \eta_{\gamma}^{\varrho}\right) \equiv 0
$$

and

$$
E_{\alpha \mid \gamma}=F_{\alpha \mid \gamma}+\frac{1}{8 \pi^{2}} \frac{\partial^{2}}{\partial x^{\alpha} \partial y^{\gamma}}\left(\int \frac{1}{L} \log (1-L) d L\right) .
$$

Equation (5.4) shows that

$$
H_{\alpha \mid \gamma}=\frac{1}{16 \pi^{2}} \frac{1}{(1-L)} \frac{\partial^{2} L}{\partial x^{\sigma} \partial y^{\varrho}}\left(\delta_{\alpha}^{\sigma} \delta_{\gamma}^{\varrho}-\eta_{\alpha}^{\sigma} \eta_{\gamma}{ }_{\gamma}\right),
$$

and in particular, $H$ contains no $\log$ terms. Equation (5.6) demonstrates that the remaining log terms contained in $G_{\alpha \mid \gamma}$ are pure gauge. This is to be expected since massless fields on $C P^{2}$ should obey Huygens principle. Therefore the integrand of (4.12) may be computed from (5.3) and (5.7).

The angular integrations in (4.12) may be done immediately to obtain

$$
S=\frac{-64}{\pi \omega_{1} \omega_{2}} \int_{-\infty}^{\infty} \int_{-\infty}^{\infty}\left(\frac{e^{i \omega_{1} b^{2} / v^{\prime}}}{v^{\prime}}\right)\left(\frac{e^{i \omega_{2} b^{2} / u^{\prime}}}{u^{\prime}}\right) G_{[\alpha, \beta] \mid[\gamma, \delta]} l_{1}^{\alpha} q_{1}^{\beta} l_{2}^{\gamma} q_{2}^{\delta} \frac{u^{\prime 2} v^{\prime 2}}{16} d u^{\prime} d v^{\prime},
$$

where

$$
\begin{array}{ll}
x^{\mu}=\frac{1}{2} \hat{k}_{1}^{\mu} v^{\prime}, & y^{\mu}=\frac{1}{2} \hat{k}_{2}^{\mu} u^{\prime} \\
\hat{k}_{1}^{\mu}=\omega_{1}^{-1} k_{i}^{\mu}, & l_{i}^{\mu}=\frac{1}{2} \hat{k}_{i}^{\mu}, \quad i=1,2 .
\end{array}
$$

Define

$$
I=\left(x \cdot y+a^{2}\right), \quad J=x^{\prime} \cdot y=\eta_{\mu v} x^{\mu} y^{v},
$$

where I am using the notation of (2.15). Then, for $x^{2}=y^{2}=0$ (since both $x^{\mu}$ and $y^{\mu}$ are null vectors):

$$
\begin{aligned}
& 4 \pi^{2} F_{[\alpha, \beta] \mid[\gamma, \delta]} x^{\alpha} q_{1}^{\beta} y^{\gamma} q_{2}^{\delta} \\
& =\frac{2 a^{2}}{\left[a^{4}(1-L)\right]^{2}}\left[1+\frac{2\left(I-a^{2}\right)}{a^{2}(1-L)}\right]\left\{( I - a ^ { 2 } ) \left[I\left(q_{1} \cdot q_{2}\right)+J \eta_{\mu \nu} x^{\mu} y^{\nu}\right.\right. \\
& \left.\left.\quad+\left(x \cdot q_{2}\right)\left(y \cdot q_{1}\right)+\left(x^{\prime} \cdot q_{2}\right)\left(y^{\prime} \cdot q_{1}\right)\right]-a^{2}\left(x \cdot q_{2}\right)\left(y \cdot q_{1}\right)\right\} \\
& \quad+\frac{4 a^{2}\left(I-a^{2}\right)}{\left[a^{4}(1-L)\right]^{3}}\left[\left(I-a^{2}\right)\left(x \cdot q_{2}\right)+J\left(x^{\prime} \cdot q_{2}\right)\right]\left[\left(I-a^{2}\right)\left(y \cdot q_{1}\right)+J\left(y^{\prime} \cdot q_{1}\right)\right],
\end{aligned}
$$




$$
\begin{aligned}
16 \pi^{2} & H_{[\alpha, \beta] \mid[\gamma, \delta]} x^{\alpha} q_{1}^{\beta} y^{\gamma} q_{2}^{\delta} \\
= & -4 / a^{2}\left(x^{\prime} \cdot q_{1}\right)\left(y^{\prime} \cdot q_{2}\right)-\frac{2}{a^{2}(1-L)}\left(q_{1} \cdot q_{2}\right) \\
& -\frac{2 a^{2}}{\left[a^{4}(1-L)\right]^{2}}\left\{2 a^{2}\left(I-a^{2}\right)\left(q_{1} \cdot q_{2}\right)-\left(I-a^{2}\right)\left(x^{\prime} \cdot q_{1}\right)\left(y^{\prime} \cdot q_{2}\right)\right. \\
& \left.+\left(y^{\prime} \cdot q_{1}\right)\left[J\left(x \cdot q_{2}\right)-\left(I-a^{2}\right)\left(x^{\prime} \cdot q_{2}\right)\right]+\left(y \cdot q_{1}\right)\left[\left(I-a^{2}\right)\left(x \cdot q_{2}\right)+J\left(x^{\prime} \cdot q_{2}\right)\right]\right\} \\
& +\frac{8 a^{4}}{\left[a^{4}(1-L)\right]^{3}}\left[\left(I-a^{2}\right)\left(x^{\prime} \cdot q_{1}\right)+J\left(y \cdot q_{1}\right)-\left(I-a^{2}\right)\left(y^{\prime} \cdot q_{1}\right)\right] \\
& \cdot\left[\left(I-a^{2}\right)\left(y^{\prime} \cdot q_{2}\right)+J\left(x \cdot q_{2}\right)-\left(I-a^{2}\right)\left(x^{\prime} \cdot q_{2}\right)\right] .
\end{aligned}
$$

Note that the first term of (5.12) has precisely the form of (5.2).

Introduce the geometric factors

$$
\begin{gathered}
A=\frac{1}{4}\left(\hat{k}_{1} \cdot \hat{k}_{2}\right), \quad B=\frac{1}{4} \eta_{\mu \nu} \hat{k}_{1}^{\mu} \hat{k}_{2}^{v}, \\
C_{i}=\frac{1}{2} \eta_{\mu \nu} \hat{k}_{i}^{\mu} f_{i}^{\nu}, \quad i=1,2 .
\end{gathered}
$$

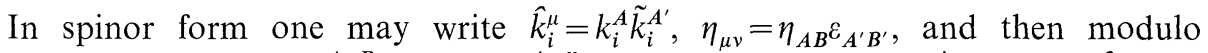
factors of $A, B \sim \eta_{A B} k_{1}^{A} k_{2}^{B}, C_{i} \sim \eta_{A B} k_{i}^{A} k_{i}^{B}$. The condition $\eta_{\mu \nu}=n_{i} \eta_{\mu \nu}^{i}$, where $n^{2}=1$ is equivalent to $\operatorname{det} \eta_{A B}=1$, which is in turn equivalent to

$$
C_{1} C_{2}=-\frac{2}{A}\left(A^{2}+B^{2}\right) \text {. }
$$

The integral (5.3) is easier to evaluate by making the change of variable

$$
u=b^{2} / v^{\prime}, \quad v=b^{2} / u^{\prime} .
$$

Then

$$
I=a^{2}+\frac{A b^{4}}{u v}, \quad J=\frac{B b^{4}}{u v}
$$

and

$$
\frac{1}{(1-L)}=-\left(\frac{a}{b}\right)^{4} \frac{u^{2} v^{2}}{[\alpha u v+\beta]},
$$

where $\alpha=2 a^{2} A, \beta=b^{4}\left(A^{2}+B^{2}\right)$.

Using this in (5.11) and (5.12), and allowing for parallel transport of $q_{1}$ and $q_{2}$ by (3.6), one gets massive cancellations which reduce the central part of the integrand of (4.12) to

$$
\begin{aligned}
& G_{[\alpha, \beta] \mid[\gamma, \delta]} l_{1}^{\alpha} q_{1}^{\beta} l_{2}^{\gamma} q_{2}^{\delta} \\
& =\frac{1}{8 \pi^{2} b^{4}}\left\{-\frac{\beta}{a^{4} A}\left(1+\varepsilon_{1}\right)\left(1+\varepsilon_{2}\right)\right. \\
& \left.\quad+\left[\left(1+\varepsilon_{1}\right)\left(1-\varepsilon_{2}\right) C_{1}^{2} v^{2}+\left(1+\varepsilon_{2}\right)\left(1-\varepsilon_{2}\right) C_{2}^{2} u^{2}\right] \frac{a^{4} A^{2} u^{3} v^{3}}{[\alpha u v+\beta]^{3}}\right\},
\end{aligned}
$$


where $\varepsilon_{i}=+1$ or -1 if $q_{i}=m_{i}$ or $\tilde{m}_{i}$ respectively. Again note that the first term is precisely the contribution arising from the topologically non-trivial zero modes of the Maxwell equations, and therefore this term is to be ignored. The remaining term manifestly preserves parity.

Substituting (5.16) into (5.8) we get

$$
\begin{aligned}
S= & \frac{-a^{4} b^{4} A^{2}}{2 \pi^{3} \omega_{1} \omega_{2}} \int_{-\infty}^{\infty} \int_{-\infty}^{\infty} e^{i \omega_{1} u} e^{i \omega_{2} v} \cdot \frac{1}{[\alpha u v+\beta]^{3}} \\
& \cdot\left[\left(1+\varepsilon_{1}\right)\left(1-\varepsilon_{2}\right) C_{1}^{2} v^{2}+\left(1+\varepsilon_{2}\right)\left(1-\varepsilon_{1}\right) C_{2}^{2} u^{2}\right] d u d v
\end{aligned}
$$

Integrating both terms twice by parts one obtains

$$
\begin{aligned}
S= & \frac{b^{4}}{16 \pi^{3}} \int_{-\infty}^{\infty} \int_{-\infty}^{\infty} e^{i \omega_{1} u} e^{i \omega_{2} v} \cdot \frac{1}{[\alpha u v+\beta]} \\
& \cdot\left[\left(1+\varepsilon_{1}\right)\left(1-\varepsilon_{2}\right) C_{1}^{2}\left(\frac{\omega_{1}}{\omega_{2}}\right)+\left(1+\varepsilon_{2}\right)\left(1-\varepsilon_{1}\right) C_{2}^{2}\left(\frac{\omega_{2}}{\omega_{1}}\right)\right] d u d v .
\end{aligned}
$$

This integral is the same as was obtained by Hawking et al. [2] in their calculations for scalar propagation. The result is that $S=0$ if $\omega_{1}$ and $\omega_{2}$ have opposite sign, and if $\omega_{1}$ and $\omega_{2}$ have the same sign then

$$
S=\frac{-b^{4}}{8 a^{2} \pi A}\left[\left(1+\varepsilon_{1}\right)\left(1-\varepsilon_{2}\right) C_{1}^{2}\left(\frac{\omega_{1}}{\omega_{2}}\right)+\left(1+\varepsilon_{2}\right)\left(1-\varepsilon_{1}\right) C_{2}^{2}\left(\frac{\omega_{2}}{\omega_{1}}\right)\right] J_{0}(p),
$$

where

$$
p^{2}=\frac{-2 b^{4}\left(A^{2}+B^{2}\right) \omega_{1} \omega_{2}}{a^{2} A} .
$$

Note that $\hat{k}_{1}^{\mu}$ and $\hat{k}_{2}^{\mu}$ are both future directed null vectors, so $A=\frac{1}{4}\left(\hat{k}_{1} \cdot \hat{k}_{2}\right)<0$, and hence $p^{2}>0$.

It should be remembered that (5.17) represents the scattering due to the secondary non-causal pole of the propagator. (The primary pole is that for causal propagation in flat space as if the $C P^{2}$ bubble was not present, and has been excluded by assuming that $\hat{k}_{1}^{\mu}$ and $\hat{k}_{2}^{\mu}$ are not parallel.) Like the results of Hawking et al. [2], this amplitude corresponds to either two particles coming in at $\mathscr{I}^{-}$and disappearing, or two particles emerging from $\mathscr{I}^{+}$with nothing going in at $\mathscr{I}^{-}$. This is just the non-causal nature of the pole manifesting itself. It does not cause physical problems, since in the foam model one has to average $S$ over positions and orientations of the bubble, and this ensures conservation of total energy and momentum.

A more detailed discussion of the causality properties of these Green's functions may be found in a forthcoming paper by Hawking.

The similarity of this work with previous foam calculations ends here. There is a glaring anomaly in (5.17) which renders it at odds with the conventional foam picture. The functional dependence of $S$ should be $J_{2}(p)$ and not $J_{0}(p)$. As it stands 
(5.17) predicts very large amplitudes for low energy scattering of spin-1 particles by gravitational bubbles.

\section{Conclusions}

The conventional viewpoint on scattering of elementary particles by foam states that for low energies the scattering amplitudes shall be of order $\left(\frac{k_{1} \cdot k_{2}}{m_{p}^{2}}\right)^{s}$. This was deduced from a collection of approximate calculations. One can also exhibit an argument based upon the WKB approximation to support this claim. My result shows that spin-1 particles scatter very strongly at low energies, indeed, the amplitude diverges as one of the incoming photon energies becomes vanishingly small. This would mean physically that if one observed a light source in a vacuum, it would be like observing it through a very dense fog. There are two possible explanations : either foam is wrong, or that the Fubini-Study metric (2.1) is not an appropriate Euclidean metric to be included in foam calculations. The latter seems more likely since $\mathscr{I}$ is highly irregular, having a non-zero shear which also diverges at one point. The Weyl tensor also does not vanish on $\mathscr{I}$. Therefore we cannot strictly analytically continue the AE metric (3.1) to a metric which is flat and Lorentzian at infinity. The curvature persists all the way out into the asymptotic region. This may well explain why one has strong scattering, it certainly explains the failure of the WKB argument supporting $S \sim\left(\frac{k_{1} \cdot k_{2}}{m_{p}^{2}}\right)$.

This paper therefore contains a cautionary tale. The indiscriminate claim that any gravitational instanton or AE metric may be used to obtain information about a Lorentzian scattering problem, is plainly dubious. Furthermore, one must be much more careful about precisely what sort of metrics one is going to consider in the Euclidean path integral (1.1), clearly one cannot include too great a contribution from (2.1).

Acknowledgement. The author is very grateful to S. W. Hawking for the many hours of time he spent discussing these results.

\section{References}

1. Hawking, S.W., Page, D.N., Pope, C.N.: The propagation of particles in spacetime foam. Phys. Lett. B86, 175 (1979)

2. Hawking, S.W., Page, D.N., Pope, C.N.: Quantum gravitational bubbles. Nucl. Phys. B170, 283 (1980)

3. Hawking, S.W.: The path integral approach to quantum gravity. In: Hawking, S.W., Israel, W. (eds.). General Relativity, Cambridge: Cambridge Univ. Press 1979

4. Freedman, M.H.: (in preparation)

5. Hitchin, N.: Compact four-dimensional Einstein manifolds. J. Diff. Geom. 9, 435 (1975)

6. Gibbons, G.W., Pope, C.N.: The positive action conjecture and asymptotically Euclidean metrics in quantum gravity, Commun. Math. Phys. 66, 267 (1979)

7. Page, D.N.: Phys. Lett. A physical picture of the K3 gravitational instanton 80B, 55 (1978)

8. Warner, N.P.: Green's functions on $C P^{n}$. Preprint, Cambridge 
9. Gibbons, G.W., Pope, C.N.: $\mathbb{C} P^{2}$ as a gravitational instanton. Commun. Math. Phys. 61, 239 (1978)

10. Gibbons, G.W., Hawking, S.W.: Classification of gravitational instanton symmetries. Commun. Math. Phys. 66, 291 (1979)

11. Gibbons, G.W., Pope, C.N.: $\mathbb{C} P^{2}$ as a gravitational instanton. Commun. Math. Phys. 61, 239 (1978)

12. Friedlander, F.G.: The wave equation on a curved space-time. Cambridge: Cambridge University Press 1975

13. Penrose, R.: Null hypersurface initial data for classical fields of arbitrary spin and for general relativity. Gen. Rel. Grav. 12, 225 (1980)

14. Pope, C.N.: Eigenfunctions and $\operatorname{spin}^{c}$ structures in $C P^{2}$. Preprint, Cambridge

Communicated by S. Hawking

Received November 30, 1981; in revised form May 20, 1982 